\title{
Rires d'initiation
}

Apprentissage et transmission des « savoir-rire »

Initiation laughters. The process of learning and transmission of "the know-

laugh"

Inès Pasqueron de Fommervault

\section{(2) OpenEdition \\ Journals}

Édition électronique

URL : https://journals.openedition.org/tc/8274

DOI : $10.4000 /$ tc. 8274

ISSN : 1952-420X

Éditeur

Éditions de l'EHESS

Édition imprimée

Date de publication : 1 décembre 2014

Pagination : 16-29

ISBN : 978-2-7351-2346-9

ISSN : 0248-6016

Référence électronique

Inès Pasqueron de Fommervault, «Rires d'initiation», Techniques \& Culture [En ligne], 62 | 2014, mis en ligne le 01 décembre 2017, consulté le 29 septembre 2022. URL : http://journals.openedition.org/tc/ 8274 ; DOl : https://doi.org/10.4000/tc.8274 


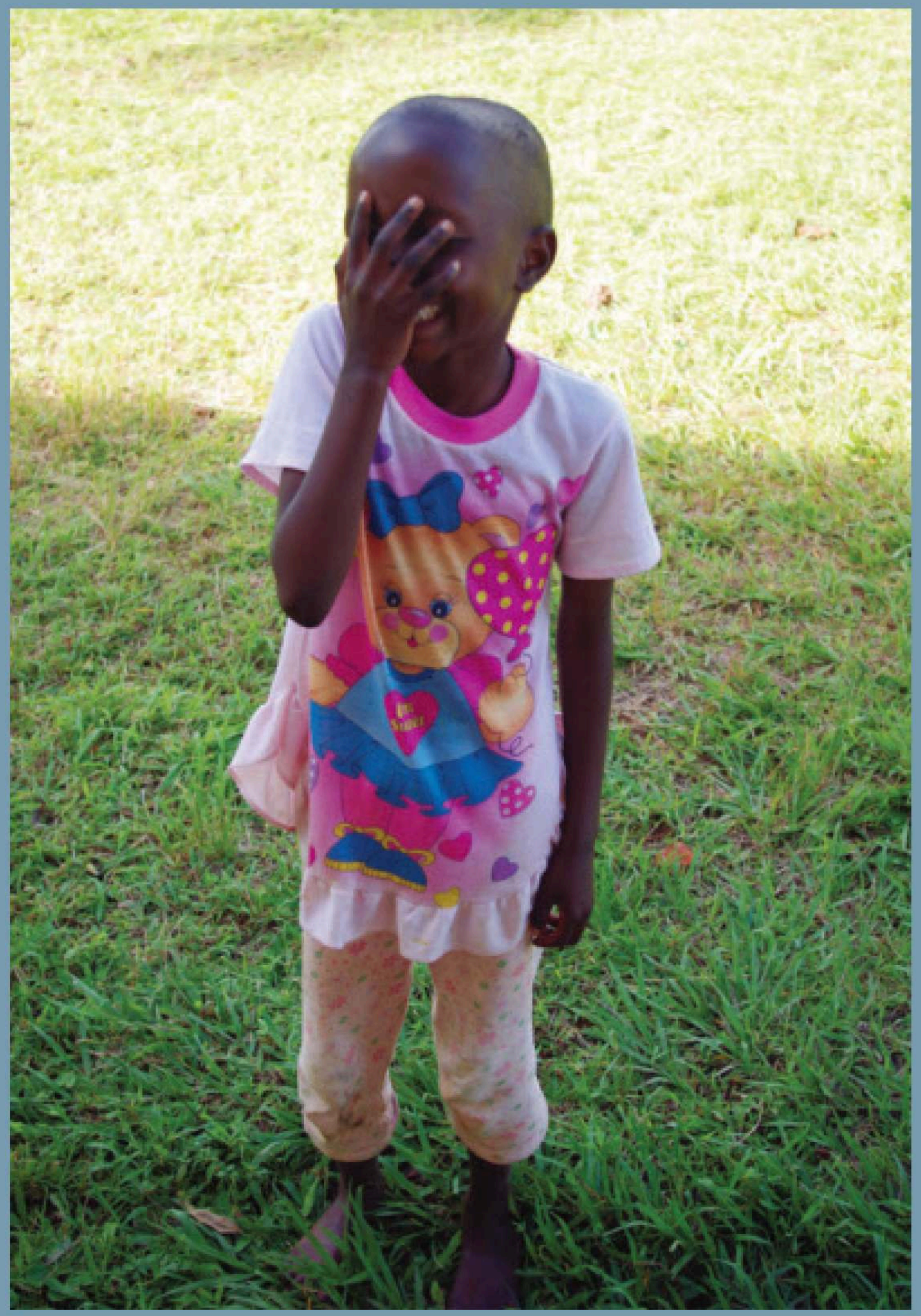




\section{RIRES D'INITIATION}

\section{Apprentissage et transmission des « savoir-rire»}

\section{Du «propre de l'homme» aux «techniques du rire»}

Dans la longue tradition humaniste et rabelaisienne (1534), le rire est appréhendé comme un signe universel et inné, parfois même mécanique (Bergson 1969). Ce langage a priori instinctif et immuable du corps est alors devenu une énième manifestation du très célèbre «propre de l'homme». Il est vrai, le rire est sans conteste une expérience universellement partagée: on ne connaît pas de société qui ne rit pas ni même d'individu incapable de rire; l'Homo sapiens est à la fois et en même temps un Homo ridens. Il n'est pas question de remettre en question ces données, néanmoins il serait réducteur de considérer seulement le rire comme un invariant de l'espèce humaine, une structure d'action fixe, sans l'insérer aussi, et surtout, dans des flux de savoirs, de valeurs et de règles dépendantes des contextes sociaux et culturels desquels ils émergent.

Dans un passage de À la recherche du temps perdu, alors que le narrateur s'interroge sur l'hérédité de la vieille noblesse française, il est soudainement interpellé par le rire du baron de Charlus: «Il eut un petit rire qui lui était spécial -un rire qui lui venait probablement de quelque grand-mère bavaroise ou lorraine, qui le tenait elle-même, tout identique, d'une aieule, de sorte qu'il sonnait ainsi, inchangé, depuis pas mal de siècles dans de vieilles petites cours de l'Europe, et qu'on goûtait sa qualité précieuse comme celle de certains instruments anciens devenus rarissimes» (Proust 1988 [1921-1922] : 332-333).

Comme Proust le suggère brillamment, il est tout à fait permis de postuler l'existence d'un mécanisme de transmission du rire au même titre que l'on admet la transmission d'une pratique instrumentale. Charlus semble avoir retenu le rire de sa grand-mère, qui 


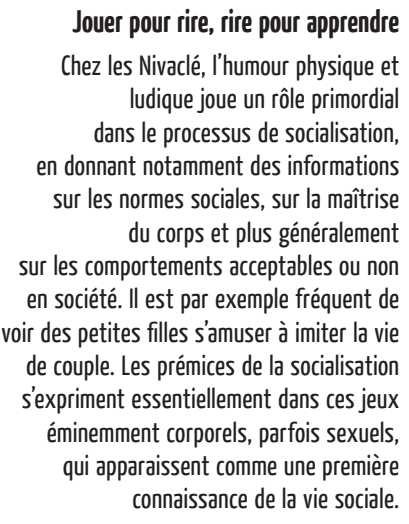

Jouer pour rire, rire pour apprendre

ungue joue un rôle primordia

en donnant notamment des informations

sur les normes sociales, sur la maîtrise du corps et plus généralement

sur les comportements acceptables ou non en société. Il est par exemple fréquent de etites filles s'amuser à imiter la vie de couple. Les prémices de la socialisation sexpriment essentiellement dans ces jeux qui apparaissent comme une première connaissance de la vie sociale.

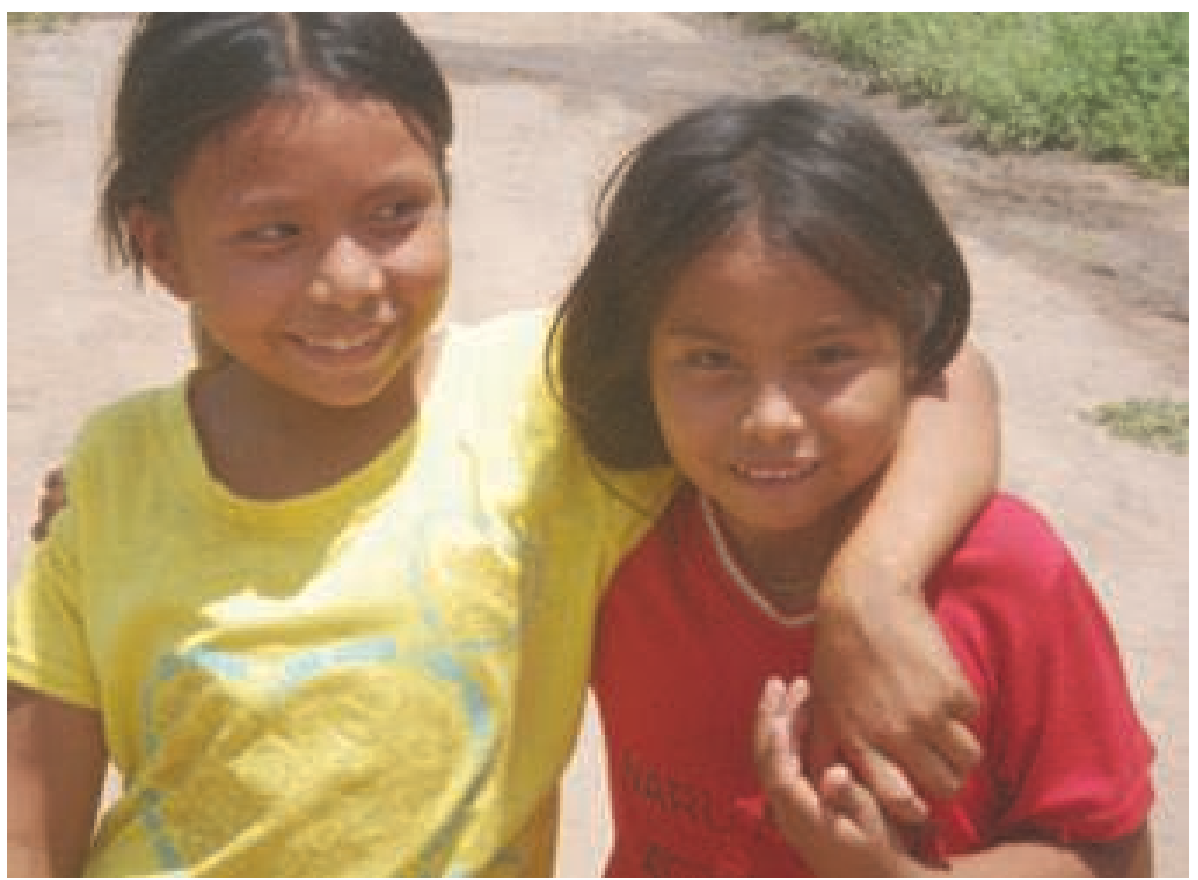

elle-même l'a appris de ses aïeules, etc. Tel un «habitus» (Bourdieu 1980), le rire s'apprend, se conserve, se diffuse, s'incorpore et s'intériorise tout au long de l'apprentissage. Véritable patrimoine immatériel, il paraît suivre une chaîne de transmission.

Or, sous prétexte de sa quotidienneté et de son apparente banalité, ces dimensions semblent gommées, comme si l'on avait abstraitement détaché l'homme qui rit de ses déterminismes sociaux et culturels. Il suffit pourtant de constater que tant que l'on ne connaît pas une société, ses normes et ses valeurs, il y a de fortes chances pour que l'on ne comprenne pas son rire, pire, qu'il nous rebute. Cette incompréhension du rire de l'Autre suffit déjà à démentir l'hypothèse d'un langage instinctif du corps. Le rire n'obéit pas seulement au contrôle strict de la génétique, il est aussi soumis à celui, non moins sévère, de la société.

Telle est précisément l'ambition de cet article, montrer que le rire est un comportement naturel, une matière brute que la société modèle et sculpte à un point tel qu'il serait pertinent de parler d'un «savoir-rire» propre à chaque société et peut-être à chaque groupe social. Montrer enfin que le rire peut être considéré comme une technique semblable aux techniques du corps tel que l'entend Marcel Mauss (1936 [1934]), à savoir une conduite sociale et corporelle dont l'apprentissage ou le «dressage», selon son expression, est conditionné par et pour l'autorité sociale.

Il s'agit ici de penser la technicité des pratiques du rire, en interrogeant sa place dans le processus d'apprentissage, notamment à travers l'exemple des «savoir-rire» des Nivaclé.

\section{Apprendre par le rire: rire d'épreuve et de maturité}

À la suite de Pierre Clastres qui, dans son fameux article «De quoi rient les Indiens?» (1974) s'était intéressé au rire des Nivaclé, j’ai à mon tour interrogé près de soixante-dix ans plus tard 
le rire de ces Indiens du Chaco paraguayen (Pasqueron de Fommervault 2013). Néanmoins, l'enjeu n'était plus de comprendre de quoi rient les Nivaclé mais plutôt comment ils rient, mettant d'emblée l'accent sur la technicité et la transmission de cette pratique quotidienne.

À la différence de l'éducation, l'apprentissage repose sur un processus d'acquisition par l'observation ou l'imitation. Il s'agit d'une éducation informelle, de savoirs quotidiens qui ne dépendent pas nécessairement d'une institution et peuvent se déployer au sein même de la famille ou du village (Rogoff \& Lave 1984). Or je postule que le rire est par excellence un moyen d'apprentissage informel. Il ne transmet pas des connaissances, mais plutôt un savoir-rire, et peut être même, un savoir-vivre. Dans une démarche ontogénétique, en exacerbant l'étroite corrélation entre le rire et l'apprentissage, j'ai voulu souligner le lien qui unit le rire et la socialisation, ce « processus par lequel la personne humaine apprend et intériorise tout au cours de sa vie les éléments socioculturels de son milieu [...] et par là s'adapte à l'environnement social où elle doit vivre» (Rocher 1970 : 132).

Pour saisir le rôle spécifique du rire dans l'apprentissage nivaclé, il me semble d'abord indispensable de soulever certains éléments caractéristiques des relations parents/enfants.

Il ne fait aucun doute que dans la plupart des sociétés la sanction physique n'est pas considérée comme amorale et est souvent utilisée au cours de l'apprentissage de l'enfant. Pourtant, aussi minime soit-il, le châtiment corporel est impensable pour ces Indiens qui l'associent à la maladie et parfois même à la mort de l'enfant.

Chez les Nivaclé, il est tout à fait inconvenant de laisser son enfant pleurer ou crier. Les larmes sont le signe d'un trouble de l'âme, lui-même perçu par analogie comme une maladie physique. Si son âme est contrariée, son corps est susceptible de devenir souffrant ${ }^{1}$. Aussi, dans l'imaginaire nivaclé, toute forme de violence parentale peut aboutir au décès de l'enfant. L'insatisfaction de l'enfant et l'incapacité à répondre à ses désirs marquent par là même le signe de l'immaturité de ses parents. Face aux pleurs de l'enfant, les parents doivent savoir user d'imagination pour le distraire afin de le maintenir dans un état de calme permanent. S'ils en sont incapables, c'est qu'ils sont irresponsables. Un parent qui punirait physiquement son enfant deviendrait nécessairement aux yeux de tous coupable de sa maladie à venir. Les parents sont donc censés accepter, comprendre et remédier à l'insatisfaction de leurs progénitures. Il est en ce sens très rare de voir quelqu'un s'insurger contre son enfant. Il semble fondamental d'appréhender l'interprétation Nivaclé des pleurs comme signe de mécontentement de l'enfant auquel on doit répondre par l'apaisement. Ce lien étroit entre les pleurs, la maladie et la mort n'est pas propre aux Nivaclé. À titre d'exemple, Françoise Héritier (1987) dans son article «Fait-on rire les enfants en Afrique?» établit une relation d'interdépendance analogue, entre les pleurs du nourrisson et la mort. Chez les Samos du Burkina Faso, le tout jeune enfant est considéré comme la réincarnation d'un ancêtre (ibid. : 65). Pour que l'ancêtre demeure dans le corps de l'enfant il faut l'empêcher de pleurer, car les pleurs interpelleraient des puissances malfaisantes, qui pourraient provoquer la fuite de l'ancêtre et conséquemment la mort de l'enfant. Chez les Samos comme chez les Nivaclé, les pleurs du nourrisson ne sont pas considérés comme naturels mais doivent absolument être apaisés, car ils seraient susceptibles de provoquer son décès.

Cette aversion pour le châtiment physique et cette inhibition des pleurs, bien qu'a priori sans rapport direct avec le sujet, voire même antagoniques, font en réalité sens dans la mesure où ce sont elles qui justifient le rôle spécifique du rire dans le processus d'apprentissage des Nivaclé.

Étant donné le refus de toute forme de violence, la menace devient une des punitions privilégiées. Parmi les figures menaçantes, il y a le renard, zorro, mais aussi C’utsjataj, un vieil homme imaginaire qui récupère les enfants désobéissants, ou encore l'infirmière 
«blanche». La menace apprend que toute infraction à la norme et aux règles aboutit fatalement à des conséquences tragiques. Mais si ces intimidations effraient les plus jeunes, elles sont une source de rire sans pareil pour les plus âgés. C'est non sans fierté que les enfants, généralement âgés de plus de six ans, affirment pouvoir en rire «parce que tout le monde sait que ça n'existe pas». Selon les Nivaclé c'est précisément à partir du moment où les enfants ne croient plus en leurs menaces et qu'ils deviennent même capables d'en rire, qu'ils peuvent être considérés comme ayant atteint le premier stade de maturité.

Cette idée pourrait être exacerbée par l'exemple du rite de la puberté. L'enfant nivaclé ayant atteint l'âge de douze ans doit procéder à tout un ensemble de rites qui se déroulent en trois étapes distinctes. La première consiste à danser et chanter à la manière des ancêtres. Le deuxième jour, l'enfant ingurgite une boisson sacrée constituée d'eau et de miel fermenté. Ce jour-là, il doit savoir boire avec modération, dans le cas contraire, il sera la cible d'insultes et de railleries, car l'on considère que c'est un signe irréfutable de son alcoolisme à venir. L'ultime étape de ce rite de puberté, celle qui précisément nous intéresse le plus dans le cadre de cet article, consiste à tester la résistance à la souffrance de l'enfant. Un groupe d'hommes attache ses jambes avec de la ficelle et pique sa cuisse avec de petits os d'animaux. Si l'enfant pleure face à cette légère douleur, il sera considéré comme immature et aura échoué à cette dernière épreuve. Inversement, si l'enfant parvient à rire ou du moins à sourire, il est considéré comme assez mature pour supporter les souffrances de la vie. Cette fête, de même que les trois étapes qui la composent, est réitérée le jour où l'individu atteint l'âge de vingt-cinq ans, le passage définitif de l'adolescence à l'âge adulte. Il devient alors impératif que le jeune adulte ne pleure pas lors du test de la souffrance, sans quoi il perdrait définitivement, aux yeux de tous, son honneur.

Même si cette fête tend aujourd'hui à disparaître elle souligne une fonction essentielle du rire chez les Nivaclé. Le rire face à la douleur ou à la peur est révélateur de la maturité d’un enfant.

Rite de maturité Nivaclé Ce rite consiste à ligoter les jambes de l'adolescent, lors du passage à l'âge adulte et de lui piquer les cuisses avec des os d'animaux. l'enfant atteste de sa maturité s'il étouffe ses pleurs et est capable de rire devant cette douleur. Le rire face à la douleur est la peur et, chez les Nivaclé, un bon moyen de mesurer la maturité d'un individu.

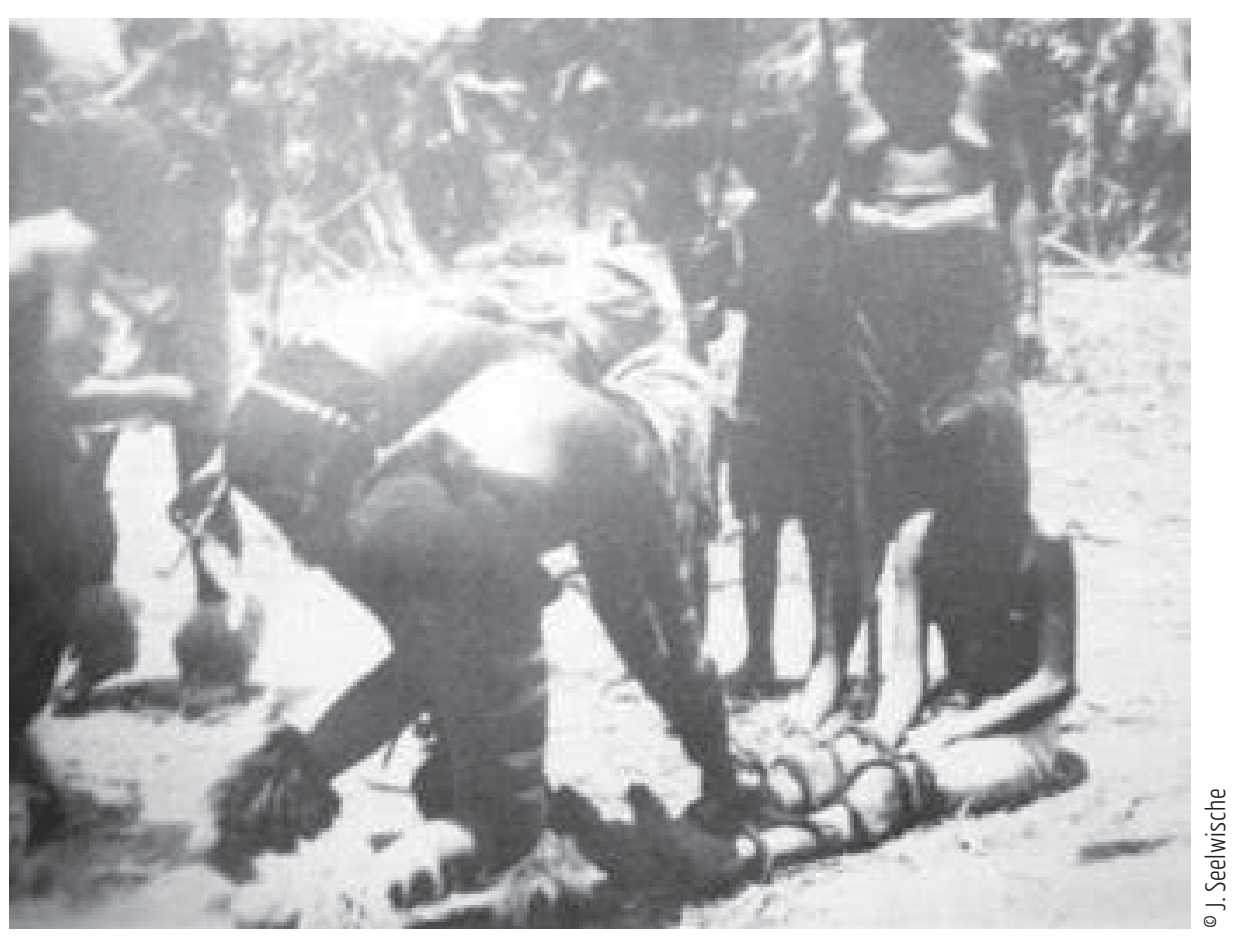


Là encore, ce lien entre le rire, l'épreuve et la maturité n'est pas propre à la société nivaclé. Il est par exemple particulièrement intéressant de noter l'existence d'une telle relation dans une société tout à fait distincte des Nivaclé, celle des Inuit. L'anthropologue Michèle Therrien a repéré une fonction éducative étonnamment similaire du rire chez les Inuit. Les parents inuit ont pour habitude de mordre leur enfant tout en leur faisant des caresses. Douceur et douleur sont étroitement mêlées et participent toutes deux à l'éducation. Cet amalgame de sensations doit être vu comme un test visant à évaluer le degré de maturité de l'enfant. Face à cette situation ambiguë, où la douleur suit le plaisir, l'enfant doit malgré tout, s'il veut prouver sa maturité, éviter d'être triste et être capable d'en rire: il s'agit de les préparer aux difficultés de la vie. Les parents inuit utilisent le rire non pas tant pour provoquer la joie de leur enfant, mais pour leur apprendre à gérer l'angoisse et la douleur. Un autre jeu fréquent consiste à dire, sans aucune véracité: « Ton père est parti à la chasse et ne reviendra pas» (Therrien 1999: 4). Si l'enfant rit devant cette blague, alors on lui confère le statut d'adulte. Chez les Inuits comme chez les Nivaclé, le rire face à l'angoisse, à la menace et à la douleur atteste à lui seul le degré de maturité d'un individu.

Ces différents exemples permettent déjà d'apercevoir le rôle non négligeable du rire dans le processus d'apprentissage. Mais chez les Nivaclé, il ne suffit pas de rire, encore faut-il savoir bien rire. C'est pourquoi il n'est pas seulement question d'«apprendre par le rire», l'essentiel est aussi et surtout d'« apprendre à rire».

\section{Apprendre à rire? La transmission des « savoir-rire»}

Dans toutes les sociétés, le corps fait l'objet d'un apprentissage, on le norme, on le corrige, on le module pour qu'il soit conforme aux valeurs et aux représentations sociales, si bien que la maîtrise du corps est intimement liée à la notion de vertu. Or le rire n'échappe pas à ce contrôle social et il est lui aussi soumis à tout un ensemble de procédures éducatives mises en place pour le discipliner. Chez les Nivaclé, le rire s'apprend et il existe des dispositifs visant à inculquer les normes d'un «savoir-rire».

Dès son plus jeune âge, l'enfant est en effet amené à contrôler et maîtriser le bon usage du rire. On lui apprend à rire au bon moment et de la bonne manière. Si, comme précédemment évoqué, les pleurs du nourrisson sont craints, certains rires sont, eux aussi, dangereux. Chez les Nivaclé, tout le monde ne peut pas rire: le rire est un droit qui sacquiert. À ce titre, le rire du nourrisson fait parti de ces rires néfastes et c'est pourquoi il est peu encouragé et même refréné. Pour rire, il est nécessaire d'être puissant et un enfant faible et fragile par nature ne doit pas rire avec excès, car il est susceptible d'y succomber. Il est courant de voir des parents réprimer le rire de leurs jeunes enfants. Une répression qu'ils justifient par le statut du nourrisson, dont le jeune âge le rend incapable de gérer l'intensité d'un rire excessif. Cette idée a d'ailleurs été confirmée par le discours tenu par un Ancien du village: «Un enfant ne doit pas rire trop. D’ailleurs, avant, nous ne faisions jamais de chatouilles à nos enfants, car on savait que ça les rendrait faibles.» Selon lui, et c'est aussi ce qu'affirment de nombreux Nivaclé, les Anciens ne cherchaient pas à faire rire leurs enfants, car ils pensaient que cela les rendrait incapables et passifs dès qu'ils atteindraient l'âge adulte. «Si on les faisait trop rire, on savait que quand ils grandiraient, ils nous demanderaient de faire tout ce qu'ils voudraient et eux ne feraient rien», ajoute-t-il. 


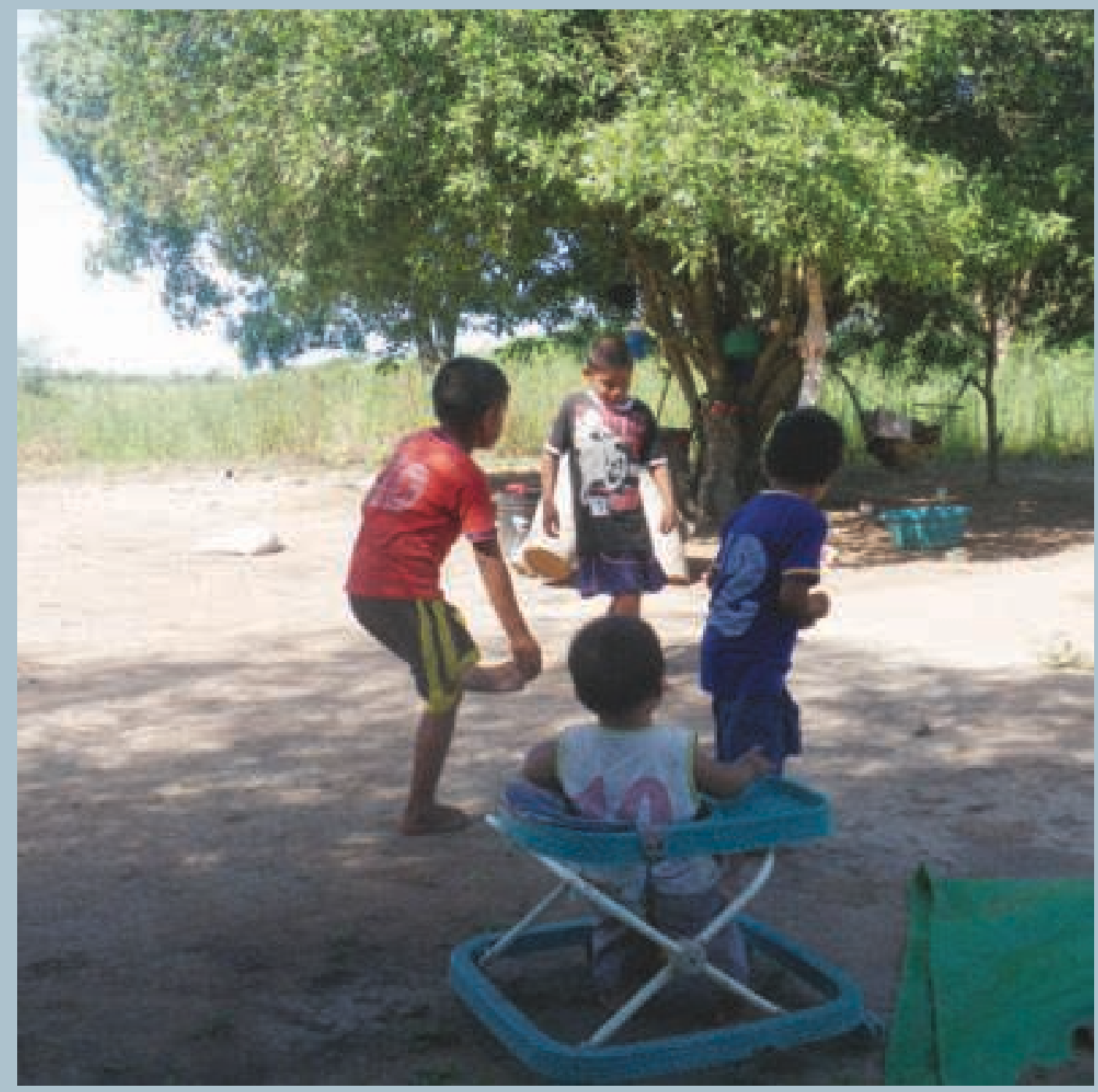

Le nourrisson, spectateur passif des interactions ludiques

Chez les Nivaclé, le rire du nourrisson n'est jamais souhaité. Les parents n'encouragent pas le rire des nouveau-nés, considérant que pour rire, il est nécessaire d'être puissant et qu'un jeune enfant, faible et fragile par nature, ne doit par conséquent pas rire avec excès. Les nourrissons sont de ce fait écartés des interactions ludiques susceptibles de stimuler leurs rires et la plupart du temps sont réduits au rôle de spectateur passif. Si le rire du nourrisson est inhibé, à partir de l'âge de 5 ans, l'enfant nivaclé est considéré comme suffisamment fort pour pouvoir rire. Dès lors les jeux, mais aussi toute forme d'humour physique ou verbal sont encouragés et même considérés comme un moyen d'apprentissage sans pareil. Bien loin d'être réprimés et refoulés, les rires infantiles sont alors véritablement exacerbés. 


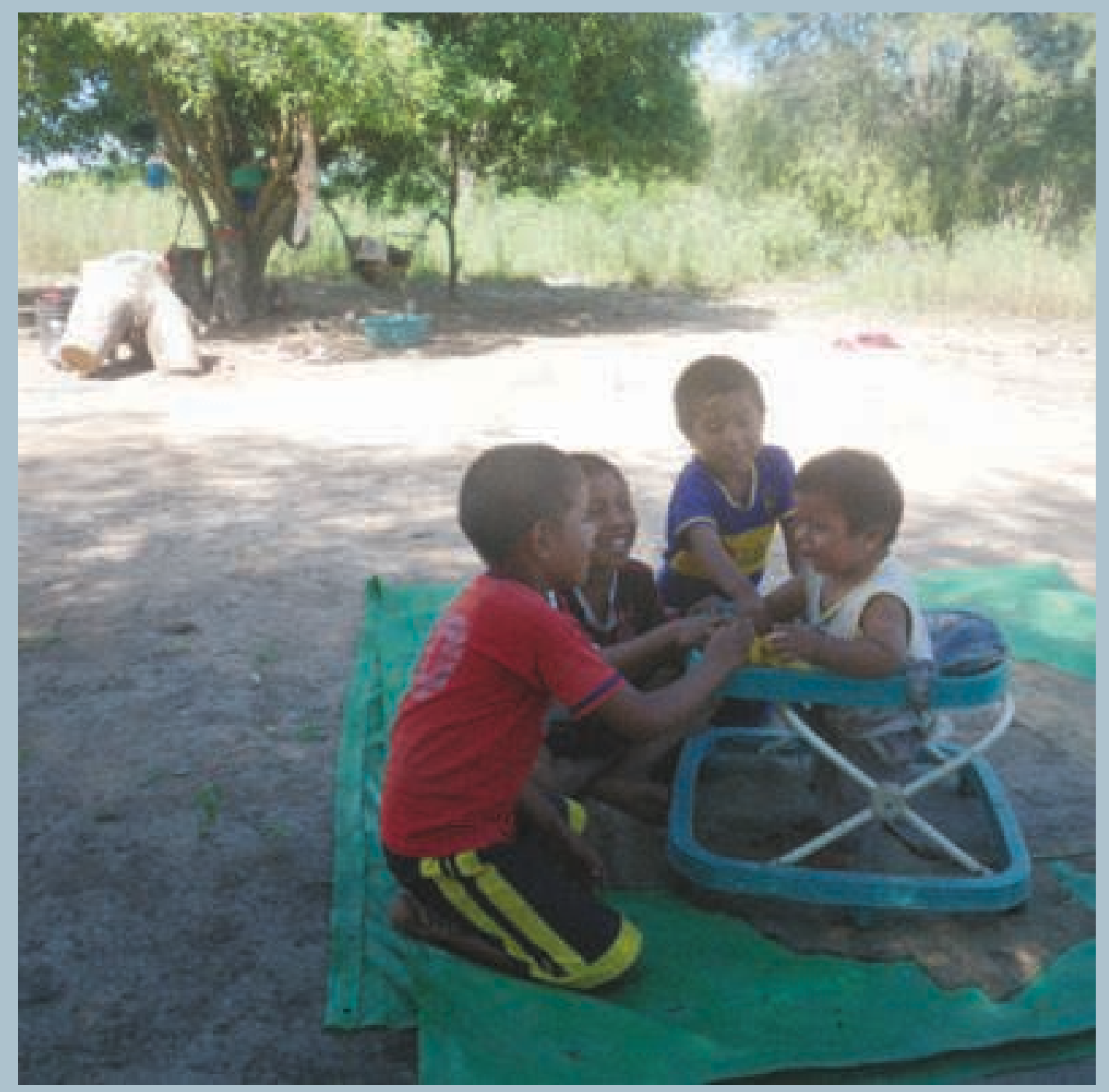

\section{Enfants nivaclés cherchant à faire rire un nourrisson}

Malgré les recommandations parentales, il arrive que les enfants plus âgés, souvent les grands frères et grandes sœurs, tentent de provoquer le rire de leur cadet, avec des grimaces et des pitreries disruptives. Ces interactions ludiques sont toutefois très rares, et généralement les enfants sont punis par les adultes témoins de la scène. Le rire du nourrisson est aussitôt refréné. 


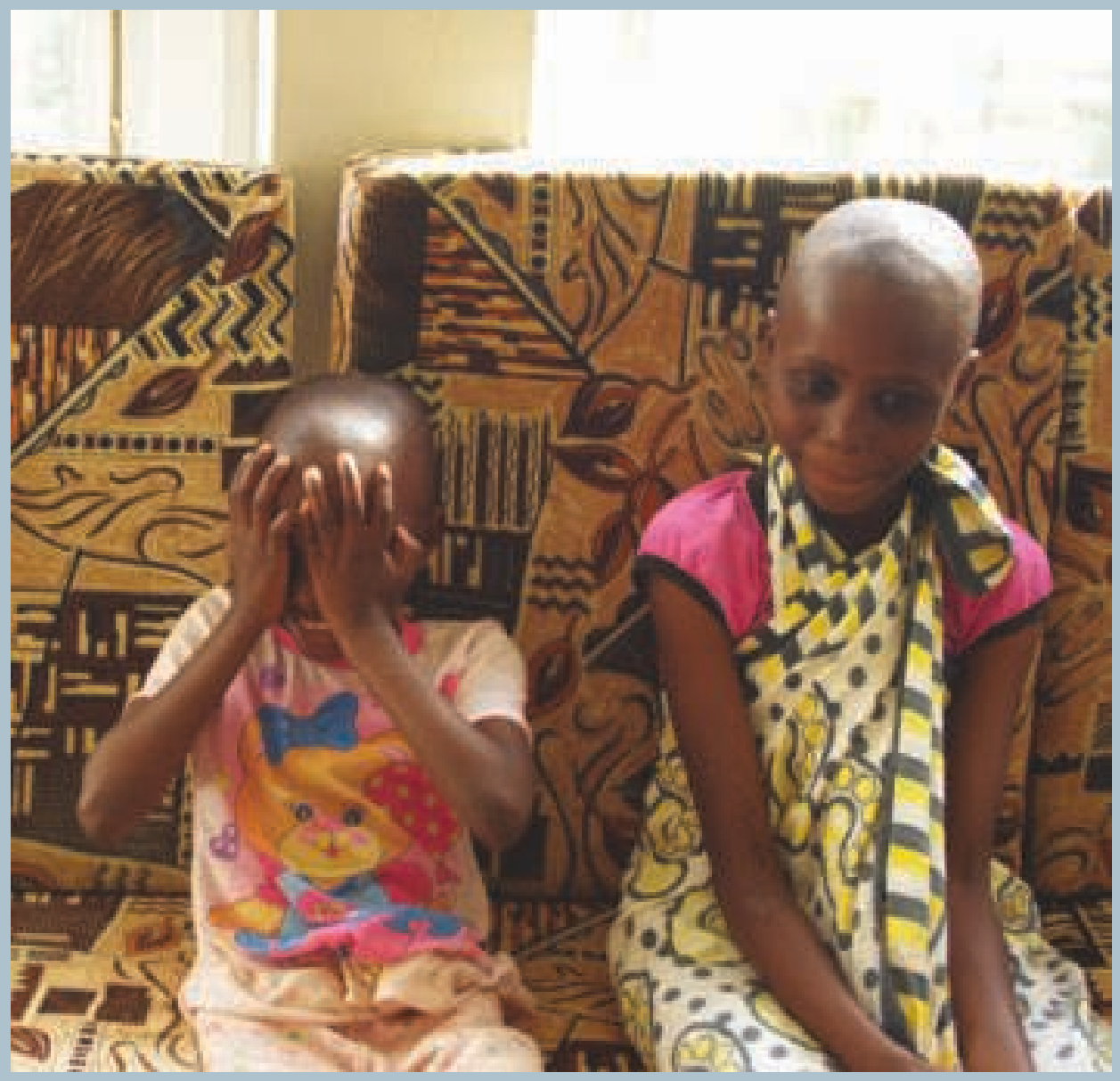

Petite fille en train de cacher son rire (Kashasha, Tanzanie)

Contrairement aux Nivaclé, les Haya de Tanzanie encouragent le rire des nourrissons en ce quiil représente une stimulation du corps et de l'esprit. Mais dès l'âge de 4-5 ans, le rire est réprimé, car il représente désormais une liberté expressive qui va à l'encontre des normes de respect et d'obéissance auxquelles sont soumis les enfants de plus de 5 ans et ce jusqu'au mariage. Le rire de l'enfant est ainsi presque toujours refoulé en public, une obligation sociale qui se manifeste par une véritable technique d'inhibition du rire: rire silencieux et main placée devant le visage. 
Pour les Anciens, le rire de l'enfant était synonyme d'oisiveté et faire rire son enfant, c'était accepter l'idée d'être et de rester dépendant de lui. Cette croyance s'est estompée avec le temps mais subsiste toujours. Il existe même un âge à partir duquel les Nivaclé considèrent que l'enfant peut rire autant qu'il le veut: entre quatre et six ans. Cet âge représente le passage du stade du nourrisson à celui d'enfant. Le changement de statut social engendre donc une modification sur l'expression et les formes du rire. À partir de cet âge, l'enfant, jusqu'ici trop faible et sujet aux maladies, est désormais considéré comme ayant suffisamment de force pour supporter l'éclat du rire. Le lien entre rire et danger qui s'observe dans ces pratiques d'inhibition est aussi présent dans l'imaginaire nivaclé; et ces représentations sociales sont à l'origine même des prescriptions et proscriptions qui entourent le rire. Je citerai à ce titre l'exemple du conte «le rire excessif et le grand feu». Ce conte de type facétieux est singulièrement révélateur du bon usage du rire en vigueur chez les Nivaclé. Son intérêt ne réside pas tant dans sa portée éminemment risible, mais dans sa capacité à exprimer en creux le comportement qu'il est nécessaire d'adopter face au rire. L'histoire déploie la terrible destinée de Ixiutax, un personnage caractérisé par son rire incessant:

«Un jour, Ixiutax, du haut de son arbre se moquait d'un groupe d'aveugles en train de se faire griller de la viande. Il décida, pour s'amuser, de leur jeter un bout de viande brûlant. Il se mit alors à rire aux éclats en voyant les aveugles paniquer. Son ami fox fox lui recouvrit la bouche pour que les aveugles ne le repèrent pas à cause de son rire. Mais un jour, Ixiutax ne put retenir et contrôler son rire et les aveugles purent savoir qui les avait brûlés. Ils lancèrent un tison en direction de ce rire qui ne s'arrêtait pas et brûlèrent Ixiutax. L'on raconte que tous les hommes échappèrent au feu qui avait pris dans la savane, excepté Ixiutax, qui, dénué de force, resta bloqué dans un nid de termites et brûla à petit feu (traduit par l'auteur)» (Johannes \& Simoneau 1988).

Ce conte expose un univers normatif dualiste particulièrement clair. Ixiutax représente le comportement déviant et immoral. Sa mort tragique sonne comme la sanction nécessaire, comme la possibilité de rétablir l'ordre qu'il avait bousculé. Apparaît ici la méfiance des Nivaclé vis-à-vis du rire. Comme je l'ai déjà évoqué, le rire démesuré fait partie des craintes parentales en ce qu'il affaiblit le corps et l'esprit du jeune enfant. L'histoire et le destin d'Ixiutax font ressurgir les méfaits d'un rire excessif et les vertus de l'art de la dissimulation et de la retenue. Ce qui se déploie ici n'est pas tant l'immoralité de Ixiutax qui se moque ouvertement des aveugles, mais le fait qu'il soit pris d'un fou rire qu'il ne parvient pas à retenir. Le mal qui ressort de ce dualisme normatif est donc sans équivoque la démesure du rire. Le rire excessif de Ixiutax représente une réalité sociale qui fait partie de l'héritage immatériel nivaclé: rire trop peut amener à sa perte. L'histoire d'Ixiutax est donc un moyen de contrôler le rire des enfants, non seulement parce qu'elle décrit les méfaits du rire incontrôlé, mais aussi parce qu'elle provoque le rire des enfants, principaux destinataires du conte, un rire maîtrisé par deux niveaux d'expérience. D'une part le contexte de transmission orale, géré par le professeur ou les Anciens (qui en sont les narrateurs) et d'autre part la structure narrative du récit. La structure du conte, comme l’ont révélé Vladimir Propp (1970) ou encore Denise Paulme (1976), faisant écho à la méthode structurale d'analyse des mythes de Claude Lévi-Strauss (1958), se fonde sur des valeurs constantes et systématiques. Il s'agit d'un temps de pause, dont le début et la fin sont déterminés à l'avance. «Le conte s'inscrit entre les formules du début, qui annoncent le "mensonge", c'est-à-dire la fiction, et celles de la fin, qui ont pour fonction le retour au monde réel. Ces formules sont là pour signifier la rupture entre deux univers, celui de la réalité celui de l'imaginaire» (Belmont 1999: 86). Le conte se présente donc comme une rupture avec la vie réelle et quotidienne. Cette 
notion d'ouverture et de fermeture des récits oraux nous permet d'appréhender le temps du conte comme structuré et contrôlé. Les contes facétieux permettent aux enfants de rire dans un cadre préétabli et institué par les plus âgés. À l'inverse du fou rire redouté, le rire suscité par la transmission orale est véritablement canalisé. Chez les Nivaclé, il subsiste donc incontestablement un apprentissage du savoir-rire qui s'apprend et se transmet par les représentations sociales et l'imaginaire nivaclé.

Dans un même ordre d'idée, Éric Jolly analyse le processus de transmission des pratiques du rire chez les Dogon. Cette transmission repose là aussi sur les imaginaires sociaux présents dans la littérature orale. Chez les Dogon, de nombreux écrits associent métaphoriquement la bouche aux orifices corporels et notamment au sexe et à l'anus. Ainsi, rire ou ouvrir la bouche peut sembler indécent dans la mesure où l'acte renvoie à l'image du sexe grand ouvert ou à l'anus béant laissant échapper des excréments, ce qui s'avère d'autant plus vrai lorsqu'il est question de rires féminins (Jolly 1999: 95). Jolly souligne ce lien étroit entre rire et obscénité à travers une histoire dogon qu'il résume ainsi: «Une veuve scandaleuse couchait avec tous les hommes de passage. Un seul homme refusait ses avances. La femme a alors préparé de la bière très alcoolisée et a invité l'homme dans l'espoir de le soûler et de le soumettre à ses désirs. L'homme est venu, mais il a tellement bu qu'il est tombé ivre mort et qu'il a déféqué en souillant ses vêtements. Quand la femme est venue le rejoindre, la nuit, elle s'est rendu compte du désastre et s'est mise à rire à rire, sans pouvoir s'arrêter. L'homme, vexé et honteux, a demandé à la femme d'aller lui puiser de l'eau pour qu'il puisse se laver et repartir discrètement. La femme est partie au puits, toujours secouée de rires, si bien qu'en se penchant elle est tombée dans le puits et s'est noyée. Quant à l'homme, ne voyant pas revenir la femme, il a fui le pays et s'est exilé pour éviter la honte et le scandale» (ibid.).

Lappétit sexuel de la femme est simultanément lié à son rire incessant et moqueur. Ce rire est finalement châtié par la perte de la veuve qui tombe maladroitement dans le puits. Outre sa dimension obscène, le caractère dangereux du rire est par là même mis en avant, puisque celle qui rit est vouée à une fin funeste.

Il apparaît donc que chez les Dogon comme chez les Nivaclé, le bon usage du rire s'apprend et se transmet et cette transmission passe notamment par les contes et la littérature orale au travers desquels le rire s'inscrit dans un système de représentations symboliques qui influe et module ses pratiques réelles et quotidiennes.

Dans certaines sociétés, l'apprentissage de ces «savoir-rire» est encore plus formalisé et le rire s'éduque presque institutionnellement. Dans son article «Le savoir-rire en Chine», l'anthropologue Hans Steinmüller montre à quel point les enfants chinois apprennent explicitement à rire (Steinmüller 2013). Il évoque l'émission TV La beauté des usages chinois destinée aux jeunes enfants et à l'apprentissage des bonnes manières. Ce programme consacre un épisode entièrement dédié au bon usage du rire ${ }^{2}$. Ce divertissement qui se déploie en un dialogue entre un élève et un Maître Sage est un réel enseignement au travers duquel l'enfant apprend à rire au bon moment et de la bonne manière selon les circonstances, le lieu, la nature de l'interaction et en fonction de son statut social. H. Steinmüller conclut qu'en Chine, l'apprentissage du rire n'est pas indépendant de celui de l'honneur, de la honte ou de toute autre façon de contenir ses émotions, si bien que «le rire et le sourire constituent une partie essentielle des usages ritualistes qui régissent la manifestation visible des émotions» (ibid. : 46). Il semble donc que le rire n'est pas seulement une extériorisation instinctive, mais qu'il est aussi une vertu sociale sans égal qui exige la maîtrise et la discipline du corps et des émotions. 
L'exemple du savoir rire Nivaclé, mis en lumière par les usages du rire chinois, ou encore les manières de rire chez les Dogons et les Samos invitent à penser le processus d'acquisition, d'apprentissage et de transmission de différents «savoir-rire» dont les prescriptions, plus ou moins explicites, plus ou moins conscientes et plus ou moins formelles, sont indissociables des valeurs et des contextes socioculturels dans lesquels ils s'inscrivent. Les acteurs sociaux doivent d'une part connaître les différents types de rire et d'autre part savoir dans quelle situation les utiliser : tout homme apprend à rire et est censé savoir rire. Autrement dit, chaque individu doit être capable d'anticiper l'interprétation de son rire par l'Autre, par rapport aux conceptions sociales et symboliques transmises par la société. Cette interprétation sous-tend au préalable la reconnaissance de différents types de rire, et plus encore, des intentions et des symboles qu'ils expriment, de sorte que rire ensemble suppose une «communauté d'interprétation» (Steinmüller 2013: 47).

\section{Dis-moi comment tu ris, je te dirai qui tu es}

Souligner le caractère social et culturel du rire, c'est en même temps prouver qu'un même comportement, tel qu'il est défini biologiquement, peut posséder des significations sociales diverses. L'acte de rire dépasse souvent le simple fait de rire, et savoir rire est une manière d'être. Bien loin d'être seulement une expression innée, rire est un savoir, une technique, peut-être même un art dont l'esthétique et la moralité s'apprennent tout au long de l'apprentissage. Tel est l'objectif de mes recherches et plus modestement de cet article: accorder une véritable place au rire dans une anthropologie soucieuse de réintégrer le banal et le quotidien dans son champ d'observation. Le rire devient alors un outil heuristique tout autant original que pertinent capable de transcender des questions fondamentales, comme celles de la nature et de la culture, de l'inné et de l'acquis.

En construisant et disciplinant l'être social, il est incontestable que le contrôle social tente de réguler et normer les comportements naturels de l'être humain. Car si le rire est inné, c'est bien techniquement qu'il s'intègre au rythme naturelde la société. Dans le rire il faut voir une technique qui résulte de la raison pratique collective. Je ferai l'hypothèse que l'apprentissage du rire est un rituel quotidien, tant il est normé et codifié; un rituel accessible aux enfants dès leur plus jeune âge et qui se présente comme un mécanisme d'apprentissage des «formes élémentaires de la vie sociale».

Si l'on acceptait l'idée d'un rituel quotidien, le rire ne pourrait plus être appréhendé indépendamment des normes sociales dans lesquelles il s'insère. Ne devrions-nous pas alors accepter qu'il varie non seulement d'une circonstance à une autre, mais aussi d'une société à une autre, d'un groupe social à un autre et peut être même d'un individu à un autre? À Jacques Le Goff de conclure: «Dis-moi si tu ris, comment tu ris, pourquoi tu ris, de qui et de quoi, avec qui et contre qui, et je te dirai qui tu es» (1997: 449). 


\section{NOTES}

Photo d'ouverture: Petite fille en train de cacher son rire (Kashasha, Tanzanie).

Toutes les illustrations sont de l'auteure sauf mention contraire.

1. Le concept de personne chez les Nivaclé possède deux dimensions, une physique et une psychique. L'essence de l'individu demeure néanmoins dans son âme, vatjeche, c'est sur elle que repose la vie et la santé de l'homme. C'est l'âme qui décide des actions du corps. Pour dire «je veux» un individu pourra dire «mon intérieur désire», et «je pense» se traduit davantage par «mon intérieur dit» (Stahl 2007: 79; Chase-Sardi 1970). L'âme et le corps sont donc inextricablement liés mais c'est l'âme qui gouverne le corps. C'est pourquoi si l'âme de l'enfant est perturbée, le corps, cach'i, deviendra à son tour malade.

2. tv.kids21.cn/index.php/video/index/13423/series/1/ plid/320.

\section{RÉFERENCES}

Bergson, H. 1969 Le rire. Essai sur la signification du comique. Paris: Broché.

Belmont, N. 1999 Poétique du conte. Essai sur le conte de tradition orale. Paris: Gallimard.

Bourdieu, P. 1980 Le sens pratique. Paris: Minuit.

Chase-Sardi, M. 1970 Cosmovisión Mak'a. En CEADUC. Suplemento Antropológico 5 (1-2).

Clastres, P. 1974 La société contre l'État. Recherches d'anthropologie politique. Paris: Minuit: chap. 6 «De quoi rient les Indiens?».

Héritier, F. 1987 «Fait-on rire les enfants en Afrique? Approche anthropologique» in M. Soulé \& B. Cramer dir. Bonjour gaieté. La génèse du rire et la gaieté du jeune enfant. Paris: ESF: 59-70.

Johannes, W. \& K. Simoneau dir. 1987 Folk Literature of the Nivaklé Indians. Los Angeles: UCLA Latin American Center Publications, University of California.

Jolly, É. 1999 «Des pleurs et des rires en pays dogon (Mali). Des lamentations pitoyables pour les morts à l'hilarité complice entre les vivants» in Du rire et des pleurs. Actes des journées Initiales 1998. Nanterre: Université Paris-X-Nanterre: 91-102.

Le Goff, J. 1997 «Une enquête sur le rire», Annales, Histoire, Sciences Sociales 52 (3) : 449-455.

Lévi-Strauss, C. 1958 Anthropologie structurale. Paris: Plon.

Mauss, M. 1935 «Les techniques du corps», Journal de Psychologie 32 (3-4): 271-293.

Pasqueron de Fommervault, I. 2013 Apprendre à rire, apprendre du rire. Le rire et l'humour dans le processus de socialisation et de construction identitaire des Nivaclé. Mémoire de Master 2 d'anthropologie, Aix-Marseille Université.

Rabelais, F. 1996 [1534] Gargantua. Paris: Points: «Avis au lecteur».

Rocher, G. 1970 [1968-1969] Introduction à la sociologie générale. Paris: Seuil.

Rogoff, B. \& J. Lave 1984 Everyday Cognition: Its Development in Social Context. Cambridge Mass.: Harvard University Press.

Paulme, D. 1976 La mère dévorante. Essai sur la morphologie des contes africains. Paris: Gallimard.

Propp, V. 1970 [1928] Morphologie du conte. Trad. fr. M. Derrida, T. Todorov \& C. Kahn, Paris: Seuil.

Proust, M. 1988 [1921-1922] À la recherche du temps perdu. Paris: Gallimard («La Pléiade») : t. III Sodome et Gomorrhe.

Stahl, W. 2007 Culturas en Interacción: Una antropología vivida en el Chaco Paraguayo. El Lector, Asuncion. Steinmüller, H. 2013 «Le savoir-rire en Chine», Terrain 61: 40-53.

Therrien, M. 1999 «Les exigences du célèbre rire inuit» in É. Daphy \& D. Rey-Hulman dir. Paroles à rire. Paris: Inalco : 211-222. 


\section{POUR CITER CET ARTICLE}

Pasqueron de Fommervault, I. 2017 «Rires d'initiation. Apprentissage et transmission des "savoir-rire" » in G. Bartholeyns \& F. Joulian, Le corps instrument, Techniques\&Culture 62: 16-29.

\section{RÉSUMÉ}

Rires d'initiation. Apprentissage et transmissions des «savoir-rire». Le rire est sans conteste une expérience universellement partagée: on ne connaît pas de société qui ne rit pas ni même d'individu incapable de rire. Il n'est pas question de remettre en cause ces données, mais de montrer que le rire ne peut pas se limiter à cette définition universaliste. L'enjeu est de mettre à jour les dimensions sociales et culturelles du rire qui, sous prétexte de sa quotidienneté et de son universalité, tendent à être gommées. Cet article invite à envisager le rire comme un savoir, peut-être même une technique, au sens maussien du terme, qui s'apprend et s'acquiert tout au long d'un processus d'apprentissage conditionné par et pour l'autorité sociale. La question de la technicité et de la transmission du rire est ici illustrée à travers l'exemple des «savoir-rire» chez les Indiens Nivaclé du Paraguay.

\section{ABSTRACT}

Initiation laughters. The process of learning and transmission of "the know-laugh». Laughing is undoubtedly a universal behavior: every humans laughs. The purpose is not to question this data but to observe the social and cultural variations of this human comportment. I suggest that there is more culture in laughter than we tend to think. The premise is simple: prove that there are social and cultural norms (explicit or unselfconscious) that affect physical laughter. This article invites to consider laughter as wisdom and perhaps a technique of the body, as understood by Marcel Mauss whose learning is conditioned by and for social authority. The question of laughter technicality and transmission will be illustrated by the example of laughter manners among the Nivaclé Indians of Paraguay.

\section{MOTS CLÉS}

Rire, technique du corps, transmission, apprentissage, savoir-faire, Nivaclé, Paraguay.

\section{KEYWORDS}

Laugher, body techniques, transmission, learning, know-how, Nivaclé, Paraguay. 